\title{
Avaliação do pH de polpas de frutas comercializadas no Município de João Pessoa, Paraíba, Brasil
}

\section{Homero Perazzo Barbosa ${ }^{1}$, Carolina Uchôa Guerra Barbosa de Lima1 ${ }^{1}$, Amanda Justino Costa ${ }^{2}$, Eduardo Uchôa Guerra Barbosa ${ }^{2}, *$ Layanna Carla Ferreira Sousa ${ }^{2}$, Marcela Carvalho César Félix ${ }^{2}$ e Kagianny Meirele Santos ${ }^{3}$}

\author{
${ }^{1}$ Faculdade de Medicina Nova Esperança. Av. Frei Galvão, 12. Gramame. João \\ Pessoa-PB, Brasil (CEP 58067-698). \\ ${ }^{2}$ Faculdade de Medicina Nova Esperança. Curso de Medicina. Av. Frei Galvão, 12. \\ Gramame. João Pessoa-PB, Brasil (CEP 58067-698). *E-mail: \\ eduardouchoa@hotmail.com. \\ ${ }^{3}$ Universidade Federal da Paraíba. Centro de Ciências Agrárias. Curso de \\ Graduação em Agronomia. Campus II. Rodovia PB-079, km 12. Areia-PB, Brasil \\ (CEP 58397-000).
}

Resumo. Neste trabalho, foi avaliado o $\mathrm{pH}$ de polpas de frutas congeladas de diferentes sabores e marcas, comercializadas no Município de João Pessoa, Estado da Paraíba, Brasil. Os sabores escolhidos foram de acerola (Malpighia emarginata Linnaeus), cajá (Spondias lutea Linnaeus), caju (Anacardium occidentale Linnaeus), goiaba (Psidium guajava Linnaeus), graviola (Annona muricata Linnaeus) e morango (Fragaria spp.) de três marcas diferentes (X, Y e Z), com a finalidade de verificar a adequação aos padrões de identidade e de qualidade para polpas de frutas estipulados pela legislação brasileira. 0 experimento foi conduzido em delineamento inteiramente casualizado (DIC), disposto em um arranjo fatorial $6 \times 3$, ou seja, seis níveis do fator sabores de polpa associados aos três níveis do fator marcas, com três repetições. Houve diferenças significativas $(\mathrm{P}<0,05)$ entre as marcas e os sabores analisados. A maior média de $\mathrm{pH}$ foi obtida para a polpa de caju $(3,67)$ e a menor para a polpa de cajá $(2,65)$. Algumas amostras (caju, graviola e morango) apresentaram valores não condizentes com o estabelecido pelo Ministério da Agricultura, Pecuária e Abastecimento, que estabelecem os parâmetros analíticos aos padrões de identidade e de qualidade para polpas de frutas.

Palavras-chave: Polpa congelada; Potencial hidrogeniônico; Qualidade; Legislação.

Abstract. pH evaluation of fruit pulps sold in the Municipality of João Pessoa, Paraíba, Brazil. In this study, it was evaluated the $\mathrm{pH}$ of frozen fruit pulps from different flavors and brands sold
Recebido

$09 / 12 / 2020$

Aceito

$10 / 04 / 2021$

Disponível on line $12 / 04 / 2021$

Publicado $30 / 04 / 2021$

Acesso aberto

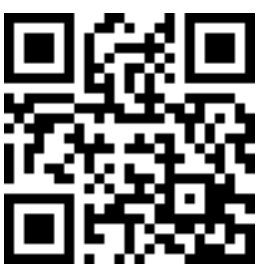

D 0000-0003-2976-4066 Homero Perazzo Barbosa

ISSN 2359-1412/RBGAS-2020-0174/2021/8/18/20/319

Rev. Bras. Gest. Amb. Sustent.

http://revista.ecogestaobrasil.net 
in the Municipality of João Pessoa, State of Paraíba, Brazil. The chosen flavors were acerola (Malpighia emarginata Linnaeus), caja (Spondias lutea Linnaeus), cashew (Anacardium occidentale Linnaeus), guava (Psidium guajava Linnaeus), soursop (Annona muricata Linnaeus) and srawberry (Fragaria spp.) from three diferente brands ( $\mathrm{X}, \mathrm{Y}$ and $\mathrm{Z}$ ) with the objective of analyze the adequacy to the standards of identity and quality for fruit pulps stipulated by Brazillian law. The experiment was conducted in fully randomized delineation (FRD), which is displayed in factorial arrangement $6 \times 3$, in another words, the 6 levels of pulp flavor factor associated with 3 levels of the brand factor, with 3 repetitions. There were significant differences $(\mathrm{P}<0.05)$ among the brands and flavors analyzed. The highest average of $\mathrm{pH}$ was obtained by the cashew pulp (3.67), and the lowest by the caja pulp (2.65). Some samples (cashew, soursop and strawberry) displayed values not in accordance with what was established by the Ministry of Agriculture, Husbandry and Supply, which set the analytic parameters of identity and quality for fruit pulps.

Keywords: Frozen pulp; Hydrogenionic potential; Quality; Law.
D) 0000-0001-9703-3156

Carolina Uchôa Guerra

Barbosa de Lima

(D) 0000-0003-1694-3188 Amanda Justino Costa

(1) 0000-0002-6818-7604

Eduardo Uchôa Guerra Barbosa

(1) 0000-0002-3276-8108 Layanna Carla Ferreira Sousa

(D) 0000-0002-5135-4664

Marcela Carvalho

César

D) 0000-0003-4120-8804

Kagianny Meirele Santos

\section{Introdução}

O consumo e a comercialização de polpa de frutas aumentam significativamente a cada ano. As pessoas estão buscando produtos saudáveis e que tenham praticidade na hora do preparo. Como as frutas são perecíveis, a polpa de fruta é uma boa opção de substituição. São produzidas nas épocas de safra, ou seja, pode-se ter matéria prima com facilidade e preço mais acessível nos períodos mais favoráveis e podem ser comercializadas de acordo com a demanda do mercado consumidor (Cáceres, 2003).

A alta perecibilidade dos frutos e sua sazonalidade impulsionam o desenvolvimento de processos tecnológicos, dentre os quais pode-se destacar a produção de polpas, que é uma atividade agroindustrial importante à medida em que agrega valor econômico à fruta, evitando desperdícios e minimizando as perdas que podem ocorrer durante a comercialização do produto in natura, além de permitir estender sua vida útil com manutenção da qualidade (Evangelista e Vieites, 2006).

Por apresentarem concentrações elevadas de açúcar simples e alta atividade de água, as frutas são importantes habitats para uma variedade de microrganismos e, portanto, são susceptíveis a uma rápida deterioração, principalmente sob conservação inadequada (Gonçalves et al., 2014; Faria et al., 2012). As infecções e intoxicações alimentares representam graves problemas de saúde pública e a transmissão de muitos patógenos, aos seres humanos, ocorre pela manipulação inadequada e/ou má conservação dos alimentos (Forsythe, 2013).

No Brasil, a qualidade das polpas de frutas comercializadas é regulamentada pelo Regulamento Técnico para Fixação dos Padrões de Identidade e Qualidade para Polpa de Frutas, contido na Instrução Normativa MAPA no 1/2000 (Brasil, 2000), pela Resolução RDC no 12/2001 (Brasil, 2001) e pela Instrução Normativa MAPA no 37/2018 (Brasil, 2018). A qualidade da polpa também está relacionada à preservação dos nutrientes e as suas características físico-químicas e sensoriais, que devem ser próximas da fruta in natura, de forma a atender às exigências do consumidor e da legislação vigente. Desse modo, aspectos como pH e outras características físicas e químicas são determinados nas normas específicas de cada tipo de polpa de fruta. 
Diante disto, o objetivo do presente trabalho foi avaliar o $\mathrm{pH}$ de diferentes polpas de frutas congeladas, comercializadas no Município de João Pessoa-PB.

\section{Material e métodos}

As amostras foram transportadas dos locais de aquisição (supermercados) em suas embalagens originais e íntegras, em caixa térmica para o Laboratório de Bioquímica das Faculdades Nova Esperança (FACENE/FAMENE), durante os meses de fevereiro e março de 2018, para posteriores análises. Como critério de inclusão, foram utilizadas polpas de frutas dentro do prazo de validade, devidamente embaladas e adquiridas no Município de João Pessoa (PB).

0 experimento foi conduzido em delineamento inteiramente casualizado (DIC), disposto em um arranjo fatorial $6 \times 3$, ou seja, seis níveis do fator sabores de polpa, três níveis do fator marcas (X, Y e Z), com três repetições totalizando 54 amostras analisadas. As polpas de frutas congeladas, das diferentes marcas, foram coletadas nos sabores de acerola (Malpighia emarginata Linnaeus), cajá (Spondias lutea Linnaeus), caju (Anacardium occidentale Linnaeus), goiaba (Psidium guajava Linnaeus), graviola (Annona muricata Linnaeus) e morango (Fragaria spp.).

Após a homogeneização, o pH das amostras foi determinado em pHmetro modelo QUIMIS Mod. Q $400 \mathrm{HM}$, previamente calibrado com soluções de pH 4,0 e 7,0, através de determinação simples e direta (Instituto Adolfo Lutz, 2008). Foram realizadas três medidas de $\mathrm{pH}$ para cada amostra, sendo o valor final dado pela média aritmética simples das medidas.

Os resultados obtidos foram comparados, também, individualmente, aos padrões estabelecidos pela normatização brasileira para cada tipo de polpa de fruta (Brasil, 2000, 2018). Para a análise de variância (Tabela 1), utilizou-se o software SAS-SYSTEM (Statistical Analysis Syste, SAS Institute Inc., North Carolina, USA) (Silva e Azevedo, 2009). Aplicou-se o teste de Tukey a 5\% de probabilidade, para comparação das médias.

\section{Resultados e discussão} Tabela 1.

O resultado da análise estatística dos dados obtidos e o Teste F são apresentados na

Tabela 1. Análise de variância dos dados experimentais.

\begin{tabular}{|l|c|c|c|c|}
\hline Fontes de Variação & GL & SQ & QM & F \\
\hline Polpas & 5 & 7,1025 & 1,4205 & $6136,58^{*}$ \\
\hline Marcas & 2 & 0,1797 & 0,0899 & $388,72^{*}$ \\
\hline Polpas x marcas & 10 & 0,1764 & 0,0176 & $76,24^{*}$ \\
\hline Erro experimental & 36 & 0,0083 & 0,00023 & - \\
\hline Total corrigido & 53 & 7,4671 & - & - \\
\hline
\end{tabular}

*Significativo a $5 \%$ de probabilidade pelo teste $\mathrm{F}$.

$\mathrm{O}$ controle de qualidade, no que diz respeito ao $\mathrm{pH}$, é muito importante na padronização do produto e na análise de alterações ocorridas durante o processamento e o armazenamento.

A Tabela 2 expressa os valores médios de $\mathrm{pH}$ das marcas e dos sabores das polpas de frutas analisadas. Houve diferenças estatisticamente significativas $(\mathrm{P}<0,05)$ entre as 
marcas e os sabores estudados, sendo que a média obtida para a marca Y foi superior às demais. A polpa de cajá foi a que apresentou menor $\mathrm{pH}(\mathrm{P}<0,05)$.

Para as amostras estudadas, os valores médios de $\mathrm{pH}$ (Tabela 2) variaram de 2,63 (cajá) a 3,79 (caju) apresentando diferenças entre si $(\mathrm{P}<0,05)$. Por sua vez, a Marca $\mathrm{Y}(\mathrm{pH}$ de 3,35) apresentou para, os diversos sabores estudados, $\mathrm{pH}$ médio superior $(\mathrm{P}<0,05)$ às outras marcas estudadas $(X=3,29$ e $Z=3,21)$. Das 18 amostras analisadas, oito não se enquadraram no padrão estabelecido.

Tabela 2. Variação do $\mathrm{pH}$ das polpas congeladas de seis sabores, em três marcas diferentes, comercializadas no Município de João Pessoa (PB).

\begin{tabular}{|c|c|c|c|c|c|}
\hline \multirow{2}{*}{ Polpas } & \multicolumn{5}{|c|}{ Marcas } \\
\hline & (X) & $(Y)$ & (Z) & Médias & Padrão* \\
\hline Acerola & $3,09 \mathrm{eA}$ & $3,07^{\mathrm{eA}}$ & $2,93^{\mathrm{eB}}$ & $3,03^{\mathrm{e}}$ & Mín. 2,8 \\
\hline Cajá & $2,66^{\mathrm{fA}}$ & $2,63^{\mathrm{fB}}$ & $2,66^{\mathrm{fA}}$ & $2,65^{f}$ & Mín. 2,2 \\
\hline Caju & $3,74^{\mathrm{aB}}$ & $3,79^{\mathrm{aA}}$ & $3,49^{b c}$ & $3,67^{\mathrm{a}}$ & Mín. 3,8 \\
\hline Goiaba & $3,68^{\mathrm{bB}}$ & $3,70^{\mathrm{bA}}$ & $3,55^{\mathrm{aC}}$ & $3,64^{b}$ & Mín. 3,5 \\
\hline Graviola & $3,43^{\mathrm{cB}}$ & $3,63^{\mathrm{cA}}$ & $3,38^{\mathrm{cc}}$ & $3,48^{c}$ & Mín. 3,5 \\
\hline Morango & $3,17 \mathrm{dC}$ & $3,26^{\mathrm{dA}}$ & $3,22^{\mathrm{dB}}$ & $3,22^{d}$ & Mín. 3,3 \\
\hline Médias & $3,29^{\mathrm{B}}$ & $3,35^{\mathrm{A}}$ & $3,21^{\mathrm{C}}$ & & \\
\hline
\end{tabular}

$\mathrm{CV}(\%)=0,46$

$\mathrm{dms}$ (polpas $/$ marcas) $=0,04$ e dms (marcas $/$ polpas) $=0,03$.

Médias seguidas da mesma letra minúscula, nas colunas, e maiúsculas, nas linhas, não diferem significativamente entre si, pelo teste de Tukey a 5\% de probabilidade.

*Instrução Normativa MAPA no 01/2000 (Brasil, 2000).

A polpa de acerola deve apresentar cor variando de amarelo ao vermelho, sabor ácido, aroma próprio e pH mínimo de 2,8 (Brasil, 2000, 2018), valor semelhante ao determinado por Canuto et al. (2010) e Lima et al. (2015). Segundo Lira Júnior et al. (2005), o pH é estabelecido como atributo de qualidade do produto pela legislação por favorecer a conservação da polpa, evitando o crescimento de microrganismos. Castro et al. (2015) encontraram pH de 3,7, para a polpa de acerola, valor mais elevado do que a média determinada neste experimento, de 3,03. Por sua vez, Santos et al. (2008) encontraram para a acerola 3,2 de pH, Bueno et al. (2002) pH de 3,3, e Oliveira et al. (1999) pH médio de 3,07. Por sua vez Brasil et al. (2016) determinaram valores de $\mathrm{pH}$ variando entre 2,84 e 3,45 . Todos os resultados encontram-se de acordo o preconizado pela legislação.

O cajá é uma fruta muito aromática e de polpa suculenta, que apresenta um sabor característico denominado de agridoce (Silva et al., 2007). Como padrão, a polpa de cajá deve ter pH mínimo de 2,2 (Brasil, 2018) valor inferior à média determinada nesta pesquisa (pH de 2,65) e próximo ao de Bueno et al. (2002) com 2,7 de pH, de Canuto et al. (2010) de 2,9 e de Oliveira et al. (1999) com média de 2,57. Por sua vez, Santos et al. (2016), avaliando diferentes marcas, determinaram valores médios superiores ( $\mathrm{pH}$ de $3,12)$.

Um estudo realizado por Caldas et al. (2010), observou que o $\mathrm{pH}$ das polpas congeladas de cajá estava de acordo com a legislação. Resultados semelhantes foram encontrados por Dantas et al. (2010), ao analisarem polpas comercializadas em Campina Grande-PB. Valores inferiores a 0,9 foram encontrados por Monção et al. (2010), onde três das cinco amostras de polpas de cajá utilizadas estavam abaixo do mínimo estabelecido. 
Lima et al. (2015) determinaram pH de 1,22 ao preconizado pela legislação. Os dados obtidos confirmam a acidez da polpa de cajá.

A polpa de caju deverá exibir cor variando do branco ao amarelado, levemente ácida e adstringente com aroma próprio. Santos et al. (2008) avaliando polpas congeladas, determinaram um $\mathrm{pH}$ de 3,2 para a polpa de caju, valor inferior à média encontrada nessa pesquisa $(3,67)$. Em outra pesquisa Santos et al. (2016), avaliando diferentes marcas, determinaram, valores médios, um pouco superiores $(\mathrm{pH}$ de 3,82$)$ porém, dentro do padrão preconizado por Brasil (2018). Canuto et al. (2010) encontraram valores de 4,7 e Oliveira et al (1999) analisando diversas amostras encontrou pH médio de 4,11. Brasil et al. (2016) determinaram valores de pH variando de 3,34 a 4,34. Dos seis sabores de polpas analisados, esta apresentou os maiores valores para $\mathrm{pH}$.

A polpa de goiaba deverá ter cor variável de branco a vermelho, sabor levemente ácido, aroma próprio e deverá atender as características determinadas e apresentar $\mathrm{pH}$ mínimo de 3,5 . 0 valor médio de pH determinado $(3,64)$ se aproxima do mínimo para aceitação para consumo e próximo ao determinado por Bueno et al. (2002) de 3,8. Evangelista e Vieites (2006) em três amostras avaliadas encontraram pH acima de 4,2; já um estudo feito por Castro et al. $(2015)$ obtiveram pH mais elevado $(4,16)$. Por sua vez, Brasil et al. (2016) determinaram valores de $\mathrm{pH}$ variando de 3,52 a 4,16.

A polpa de graviola deve mostrar cor variando do branco ao marfim, sabor ácido, aroma próprio e pH mínimo de 3,5 (Brasil, 2018), valor um pouco superior ao do presente trabalho de 3,48. Por sua vez, Canuto et al. (2010) determinaram pH de 3,7. Os valores de pH, para polpa de graviola, determinados por Leal et al. (2013) estavam fora dos padrões de identidade e qualidade (PIQ) estabelecidos.

Siqueira et al. (2009) encontraram valores médios $(\mathrm{pH} 3,75)$ para polpa de morango conservadas em refrigeração. $\mathrm{O}$ pH de três marcas de polpas de morango congeladas, variaram entre 3,50 e 3,61 em estudo realizado por Pereira et al. (2006). Por sua vez, Oliveira et al. (2012) encontraram valor de 3,60. Os valores obtidos, nesta pesquisa, para polpa de morango, estão abaixo dos padrões de identidade e qualidade (PIQ) estabelecidos (pH de 3,30) de acordo com a Instrução Normativa MAPA no 37/2018 (Brasil, 2018).

Fatores edafoclimáticos, o tempo de maturação e o processamento da fruta, podem influenciar na variação de $\mathrm{pH}$ das polpas das frutas. A presença de ácidos orgânicos, componentes importantes na formação de diversas propriedades das frutas, também pode contribuir para a variação do pH (Santos et al., 2014).

Segundo Castro et al. (2015), para a preservação dos alimentos, a indústria tira proveito da ação do $\mathrm{pH}$ sobre os microrganismos, pois o baixo $\mathrm{pH}$ diminui a microbiota alterante. Dessa forma, as polpas de frutas devem apresentar $\mathrm{pH}$ abaixo de 4,5 para que sejam conservadas de maneira adequada sem necessidade do uso de altas temperaturas e que assim não coloque em risco sua qualidade.

\section{Conclusão}

Após as análises de $\mathrm{pH}$, constatou-se diferenças entre as marcas e os sabores das polpas de frutas analisadas e que há a necessidade de adequação para atendimento dos Padrões de Identidade e Qualidade (PIQ) vigentes e estipulados pela legislação. As marcas $\mathrm{X}, \mathrm{Y}$ e Z apresentaram, respectivamente, $50 \%, 33 \%$ e $50 \%$ valores de $\mathrm{pH}$ fora do padrão estabelecido pela legislação vigente, devido as alterações do pH nas polpas caju, graviola e de morango. Por sua vez, as polpas de acerola, cajá e de goiaba estavam de acordo com a legislação. As variações encontradas nos resultados provavelmente podem ser falhas no processo produtivo das polpas de frutas indicam que há a necessidade de padronização do processo tecnológico para a obtenção de polpas de frutas de qualidade. 


\section{Referências}

Brasil, A. S.; Sigarini, K. S.; Padrinho, F. C.; Faria, R. A. P.; Siqueira, N. F. M. P. Avaliação da qualidade físico-química de polpas de frutas congeladas comercializadas na Cidade de Cuiabá/MT. Revista Brasileira de Fruticultura, v. 38, n. 1, p.167-175, 2016. https://doi.org/10.1590/0100-2945-253/14

Brasil. Instrução Normativa MAPA no 01, de 07 de janeiro de 2000. Aprova o Regulamento Técnico Geral para Fixação dos Padrões de Identidade e Qualidade para Polpa de Fruta. Disponível em: <https://www.gov.br/agricultura/ptbr/assuntos/vigilancia-agropecuaria/ivegetal/bebidas-arquivos/in-no-1-de-7-de-janeirode-2000.doc>. Acesso em: 27 nov. 2020.

Brasil. Resolução RDC no 12, de 2 de janeiro de 2001. Disponível em: <http://bvsms.saude.gov.br/bvs/saudelegis/anvisa/2001/res0012_02_01_2001.html>. Acesso em: 27 nov. 2020.

Brasil. Instrução Normativa MAPA no 37 , de 1 de outubro de 2018. Disponível em: $<$ https://www.gov.br/agricultura/pt-br/assuntos/inspecao/produtos-vegetal/legislacao1/biblioteca-de-normas-vinhos-e-bebidas/instrucao-normativa-no-37-de-1o-de-outubrode-2018.pdf>. Acesso em: 27 nov. 2020.

Bueno, S. M.; Lopes, M. R. V; Graciano, R. A. S; Fernandes, E. C. B. F.; Garcia-Cruz, C. H. Avaliação da qualidade de polpas de frutas congeladas. Revista do Instituto Adolfo Lutz, v. 62, n. 2, p. 121-126, 2002.

Cáceres, M. C. Estudo do processamento e avaliação da estabilidade do blend misto a base de polpa de tamarindo (Tamarindus indica L.) e uso de beterraba (Beta vulgaris). Campinas: Universidade Estadual de Campinas, 2003. (Dissertação de mestrado).

Caldas, Z. T. C.; Araújo, F. M. M. C.; Machado, A. V.; Almeida, A. K. L.; Alves, F. M. S. Investigação de qualidade das polpas de frutas congeladas comercializadas nos Estados da Paraíba e Rio Grande do Norte. Revista Verde de Agroecologia e Desenvolvimento Sustentável, v. 5, n. 4, p. 156-163, 2010.

Canuto, G. A. B.; Xavier, A. A. D.; Neves, L. C.; Benassi, M. T. Caracterização físico-química de polpas de frutos da Amazônia e sua correlação com a atividade anti-radical livre. Revista $\begin{array}{lllll}\text { Brasileira de Fruticultura, } & \text { v. 32, } & \text { n. } 4, & \text { p. 1196-1205, } 2010 .\end{array}$ https://doi.org/10.1590/S0100-29452010005000122

Castro, T. M. N.; Zamboni, P. V.; Dovadoni, S.; Cunha Neto, A.; Rodrigues, L. J. Parâmetros de qualidade de polpas de frutas congeladas. Revista do Instituto Adolfo Lutz, v. 74, n. 4, p. 426-436, 2015.

Dantas, R. D. L.; Rocha, A. P. T.; Araújo, A. D. S.; Rodrigues, M. D. S. A.; Maranhão, T. K. L. Perfil da qualidade de polpas de fruta comercializadas na Cidade de Campina Grande/PB. Revista Verde de Agroecologia e Desenvolvimento Sustentável, v. 5, n. 5, p. 61-66, 2010.

Evangelista, R. M.; Vieites, R. L. Avaliação da qualidade de polpa de goiaba congelada, comercializada na Cidade de São Paulo. Segurança Alimentar e Nutricional, v. 13, n. 2, p. 76-81, 2006. https://doi.org/10.20396/san.v13i2.1834

Faria, M.; Oliveira, L. B. D.; Costa, F. E. C. Qualidade microbiológica de polpas de açaí

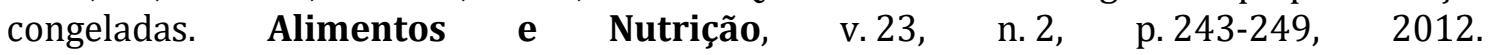
https://doi.org/10.34117/bjdv6n3-182 
Forsythe, S. J. Microbiologia da segurança dos alimentos. 2. ed. São Paulo: Artmed, 2013.

Gonçalves, M. V. V. A.; Silva, J. P. L.; Rosenthal, A.; Furtado, A. S. L.; Calado, V. M. A. Incidência de fungos termorresistentes e propriedades microbiológicas da polpa de cupuaçu congelada (Theobroma grandiflorum Schum). Perspectivas Online: Biológicas \& Saúde, v. 14, n. 4, p. 41-49, 2014. https://doi.org/10.25242/8868414201452

Instituto Adolfo Lutz. Métodos físico-químicos para análise de alimentos. 1. ed. São Paulo: Instituto Adolfo Lutz, 2008.

Leal, R. C.; Reis, V. B.; Luz, D. A. Avaliação de parâmetros físico-químicos de polpas congeladas de graviola comercializadas em supermercados de São Luiz-MA. Cadernos de Pesquisa, v. 20, n. 2, p. 76-80, 2013. https://doi.org/10.18764/2178-2229.v20n2p76-80

Lima, T. L. S.; Cavalcante, C. L.; Sousa, D. G.; Silva, P. H. A.; Andrade Sobrinho, L. G. Avaliação da composição físico-química de polpas de frutas comercializadas em cinco cidades do Alto Sertão paraibano. Revista Verde de Agroecologia e Desenvolvimento Sustentável, v. 10, n. 2, p. 49-55, 2015.

Lira Júnior, J. S.; Musser, R. S.; Melo, E. A., Maciel, M. I. S.; Lendrman, I. E.; Santos, V. F. Caracterização física e físico-química de frutos de cajá-umbu (Spondias spp.). Food Science and Technology, v. 25, n. 4, p. 757-761, 2005.

Monção, E. D. C.; Silva, E. F.; Sousa, P. B.; Silva, M. D. J.; Sousa, M. M. Avaliação físico-química e centesimal de polpas congeladas de cajá (Spondias lutea L.) e de manga (Mangifera indica L.) consumidas em Teresina-PI. Anais do Congresso de Pesquisa e Inovação da Rede Norte Nordeste de Educação Tecnológica - CONNEPI 2010, Maceió, 2010.

Oliveira, M. E. B.; Bastos, M. S. R.; Feitosa, T.; Branco, M. A. A. C.; Silva, M. G. G. Avaliação de parâmetros de qualidade físico-químicos de polpas congeladas de acerola, cajá e caju. $\begin{array}{llllll}\text { Ciência e Tecnologia de Alimentos, } & \text { v. 19, } & \text { n. 3, p. 326-332, } 1999 .\end{array}$ https://doi.org/10.1590/S0101-20612005000400021

Oliveira, R. C.; Rossi, R. M.; Barros, S. T. D. Estudo reológico da polpa de morango (Fragaria vesca) em diferentes temperaturas. Acta Scientiarum. Technology, v. 34, n. 3, p. 283-288, 2012. https://doi.org/10.4025/actascitechnol.v34i3.7833

Pereira, J. M. A. T.; Oliveira, K. A. M.; Soares, N. F. F.; Gonçalves, M. P. J. C.; Pinto, C. L. O.; Fontes, E. A. F. Avaliação da qualidade físico-química, microbiológica e microscópica de polpas de frutas congeladas e comercializadas na Cidade de Viçosa-MG. Alimentos e Nutrição, v. 17, n. 4, p. 437-442, 2006.

Santos, C. A. A.; Coelho, A. F. S; Carreiro, S. C. Avaliação microbiológica de polpas de frutas congeladas. Ciência e Tecnologia de Alimentos, v. 28, n. 4, p.913-915, 2008. https://doi.org/10.1590/S0101-20612008000400023

Santos, E. H.F.; Figueiredo Neto, A.; Donzeli, V.P. Aspectos físico-químicos e microbiológicos de polpas de frutas comercializadas em Petrolina (PE) e Juazeiro (BA). Brazilian Journal of Food Technology, v. 19, e2015089, 2016. https://doi.org/10.1590/1981-6723.8915

Santos, J. S.; Santos, M. L. P.; Azevedo, A. S. Validação de um método para determinação simultânea de quatro ácidos orgânicos por cromatografia líquida de alta eficiência em polpas de frutas congeladas. Química Nova, v. 37, n. 3, p. 540-544, 2014. https://doi.org/10.5935/0100-4042.20140087 
Silva, F. A. Z.; Azevedo, C. A. V. Principal components analysis in the software assistat: Statistical attendance. World Congress on Computers in Agriculture, American Society of Agricultural and Biological Engineers, 2009.

Silva, Y. C.; Mata, M. E. R. M. C.; Duarte, M. E. M.; Cavalcanti, A. S. R. R. M.; Oliveira, C. C. A.; Guedes, M. A. Análise sensorial da polpa e do suco de cajá obtidos pela reidratação de cajá em pó. Revista Brasileira de Produtos Agroindustriais, v. 9, n. 1, p. 1-6, 2007. https://doi.org/10.15871/1517-8595/rbpa.v9n1p1-6

Siqueira, H. H.; Vilas Boas, B. M.; Silva, J. D.; Nunes, E. E.; Lima, L. C. O.; Santana, M. T. A. Armazenamento de morango sob atmosfera modificada e refrigeração. Ciência e Agrotecnologia, v. 33, n. esp., p. 1712-1715, 2009. https://doi.org/10.1590/S141370542009000700002

Informação da Licença: Este é um artigo Open Access distribuído sob os termos da Licença Creative Commons Attribution, que permite uso irrestrito, distribuição e reprodução em qualquer meio, desde que a obra original seja devidamente citada. 